\title{
HIV and surgeons
}

\author{
The risks are small
}

The complex ethical and practical issues surrounding the question of preoperative testing for HIV antibodies in patients before surgery have been fuelled by a report from the Royal College of Surgeons of Edinburgh that advocated testing for those people suspected of being infected with HIV - normally with consent but without it in emergencies. ${ }^{1}$ The issues include the degree of risk to health care workers, whether this risk is reduced by prior knowledge of the HIV status of the patient, the utility of adopting "universal precautions," and the advantages and disadvantages to the patient of HIV testing. The Royal College of Surgeons of England was due to make a statement on the subject last week but postponed it at the last minute for further discussion.

The risk to the surgeon of becoming infected with HIV as a result of a sharps injury is small. Those most at risk seem to be nurses, and there is circumstantial evidence in only four surgeons that occupational exposure might have resulted in seroconversion. ${ }^{3}$ The risk of seroconversion has been calculated to be one infection every eight years in high prevalence $(25-30 \%)$ areas given a case load of 15000 patients a year $^{4}$ and as small as one infection every 80 years in low prevalence $(<3 \%)$ areas. ${ }^{5}$ Nevertheless, HIV infection is a devastating disease that ends in death; though the fear it engenders among health workers is out of proportion to the risks, ${ }^{6}$ surgeons and nurses may be forgiven for an emotional response to the dangers.

Most surgeons think that if they know the HIV status of a patient they will both be more careful and adopt different techniques, but with the low infection rate scientific confirmation of this is probably impossible. One study from San Francisco that examined this issue showed that on average surgeons contaminate themselves with blood in $8.7 \%$ of cases and have a penetrating injury in $1.7 \%$. $^{4}$ These risks were increased during operations lasting more than three hours or in which more than $300 \mathrm{ml}$ of blood was lost but were unaltered by knowledge of the HIV status of the patient. This study should be evaluated with caution because the surgeons, working in a high prevalence area, were already taking maximum care. They were also using universal precautions.

There is no scientific evidence that "universal precautions" reduce the risk of HIV infection. As five cases of seroconversion have occurred after massive exposure of the skin or mucous membranes to blood, ${ }^{3}$ it would seem sensible to wear an impervious gown during surgery. Wearing of goggles or visors is suggested as there is a theoretical risk of HIV in aerosols being transferred through the conjunctiva. This has been a particular worry recently in orthopaedic surgery, but the low occupational risks of dentists, who are heavily exposed to aerosols, is reassuring. ${ }^{7}$ Double gloving undoubtedly reduces the risk of contamination of the hands with blood and probably the number of needlestick injuries. ${ }^{4}$

More widespread use of HIV antibody testing has been advocated recently ${ }^{8}$ as a result of studies showing that antiretroviral treatment ${ }^{9}$ or prophylaxis against Pneumocystis carinii pneumonia ${ }^{10}$ in asymptomatic people may improve the prognosis. Testing before an operation may not, however, be the best environment for counselling, and surgeons would have to become conversant with the many complex problems that this raises. There are also clear disadvantages to the patient in preoperative HIV testing. Confidentiality is a particular problem when the "need to know" principle applies to large numbers of staff. High risk patients tend to be placed last on the operating list, where their operation is most likely to be cancelled. The alternative possibility that has been suggested - transfer to a "high risk" centre ${ }^{1}$ - is likely to be unpopular. It also places an unfair burden on staff working in such units as there is no evidence that any extra measures they may take will provide increased protection.

An important concern about widespread preoperative testing has been the incidence of false positive results, ${ }^{11}$ but these should be reduced to zero by using the most recently available methods and confirming all positive findings with a second sample.

No surgical group in Britain is advocating universal screening preoperatively-only that "high risk" groups should be tested. The assessment of high risk groups, however, particularly in women, is likely to be inaccurate, ${ }^{12} 13$ and the criteria used will often be pejorative. Nevertheless, in practice, attempts to define two tiers of risk with the help of HIV antibody testing with consent are likely to continue because in areas of low prevalence and low risk (most of Britain) universal precautions are not necessary. There are changes in the practice of surgery that can be undertaken without disadvantage, however, and these include not handling sharps during operations, not resheathing needles, and disposing of sharps carefully. There are also practices that are plainly illogical, and these include automatically placing high risk patients at the end of the list. If a patient is known to 
be HIV positive then surgeons and other health care workers will accept the added inconvenience and possible disadvantages of an impervious gown, double gloving, and eye protection.

This two tier system of precautions becomes controversial when applied to people thought to be in high risk groups. These judgments are not disinterested. Decisions about high risk behaviour and HIV testing will potentially benefit the staff as well as the patient, so they raise difficult ethical issues that require full discussion at an early stage and an appreciation that special counselling skills may be required. Many patients who perceive themselves to be at risk will agree to be tested because of potential benefits to themselves or theoretical protection for staff. It must be made clear to those patients who decide not to be tested, however, that clinical decisions about their management will be unaffected.

B G GAZZARD

Consultant physician

C WASTELL

Westminster Hospital,

Professor of surgery
1 Roval College of Surgeons of Edinhurgh. Statement to fellows on HIV infection and AIDS Edinhurgh: Roval College of Surgeons of Edinburgh, 1989.

2 Centre for Disease Control. Recommendations for prevention of HIV transmission in health care settings. M.MWR $1987: 36$ suppl $2 S$

3 Centre for Disease Control. 1989 guidelines for prevention of transmission of human immunodeficiency virus and hepatitis $\mathrm{B}$ virus to health care and public safety workers. $M M W R$ 1989:38 suppl S6

+ Gerberding JL. Littell C, Tarkington A, Brown A, Schecter WP. Risk of exposure of surgical personnel to patients' blood during surgery at San Francisco General Hospital. N Engl f Med 1990;322:1788-93.

5 Leentvar-Kuijpers A. Keeman JN, Dekker E, Dekker MM, Ansink-Schipper MC, Coutinho RA. HIV: occupational risk of surgical specialists and operating room personnel in the St Lucas Hospital in Amsterdam. Ned Tijdschr Geneeskd 1989:133:238-91.

6 Gerbert B, Maguire B, Badenaar V, Aitman D, Stone G. Fear of AIDS: issues for health professional education. AIDS Education and Prevention 1989:1:39-52.

Klein RS, Fehelan JA, Freeman K, $t$ al. Low occupational health risk of human immunodeficiency virus infection amongst dental professionals. N Engl f Med 1988:318:86-90.

8 Health and Public Policy Committee of the American College of Physicians. The acquired immunodeficiency syndrome and infection with the human immunodeficiency virus. Ann Intern

9 Volberding PA. Lagakos SW, Koch MA, et al. Zidovudine in asymptomatic human immunodeficiency infection-a controlled trial in persons with fewer than $500 \mathrm{CD} 4$-positive cells per cubic millimeter. N Engl f Med 1990;322:941-9.

10 Fishel MA, Dickinson GM, La Voie L. Safety and efficacy of sulphamethoxazone and trimethoprim chemoprophylaxis for Pneumocystis carinii pneumonia in AIDS. FAMA 1988 259:1185-9.

11 Hagen MD, Klemens B, Meyer MD, Pauker SG. Routine preoperative screening for HIV: Does the risk to the surgeon outweigh the risk to the patient? FAMA 1988;259:1357-9.

12 Landesman S, Minkoff H, Holman S, McCalla S, Sijin O. Serosurvey of human immunodeficiency virus infection in parturients: implications for human immunodeficiency virus testing programs of pregnant women. FA.HA 1987;258:2701-3.

13 Krasinki K. Borkowsky W, Bebenroth D, Moore T. Failure of voluntary testing for human immunodeficiency virus to identify infected parturient women in a high-risk population. $N$ Engl 7 Med 1988:318:185.

\section{Prospects for preventing heart disease}

\section{Population based approaches offer a cost effective way of reducing risk}

Coronary heart disease remains the principal cause of death in many economically advanced countries despite a decline in morbidity and mortality in the past two decades of up to a half in the United States, Australia, Canada, and New Zealand. ${ }^{12}$ The decline has been greatest among the socially advantaged and the young. ${ }^{3}$ The fall in morbidity and mortality has paralleled changes in risk factor levels - often poorly documented-and improvements in coronary care, ${ }^{12}$ and a causal relation seems likely. ${ }^{+}$For almost 40 years markers for risk of coronary heart disease have been known, including personal attributes such as high blood pressure, high total serum cholesterol concentration, and cigarette smoking. What is now also known is that levels of these risk factors and the prevalence of coronary heart disease vary within a given population on the basis of socioeconomic status 5 and that these variations exist even in prepubescent children. ${ }^{6}$

Risk factors for coronary heart disease measured and applied to groups have a limited predictive power for future coronary events in those groups. About half of the variance in most population risk equations remains unexplained. ${ }^{2}$ The predictive value of risk factors is even lower in individuals, yet measurement of risk factors is commonly a part of medical treatment aimed at reducing individual risk. The results from several randomised multiple risk factor intervention trials have altered perceptions of the value of intervention. Population based interventions aimed at dietary or smoking habits, or both, may lower levels of risk factors and the incidence of coronary heart disease,${ }^{8}$ although such studies have their critics. $^{9}$

The medical profession's response to preventing coronary heart disease is based on several factors. Firstly, identifying people at high risk is attractive when, as with hypertension, effective and increasingly acceptable (but expensive) medical treatment is available. Secondly, difficulty in helping people to change their lifestyles, especially diet and smoking, when society actively promotes the consumption of cigarettes and convenience foods has been (and continues to be) a source of dejection among doctors. Thirdly, forces that affect the community's health, such as societal attitudes, institutionalised promotion of unhealthy lifestyles and products, and social disadvantage, have often been judged as being beyond the professional concern of doctors. As a result medical effort has been concentrated on those at high risk. If applied to the management of cholesterol concentration this approach will lead to an expensive phase of medical care as so many people in technologically advanced societies have cholesterol concentrations that place them at increased risk of coronary heart disease. Furthermore, such an approach is less likely to reach those in most need - the socially disadvantaged. ${ }^{10}$

An alternative approach, which is not incompatible with clinical intervention for people at high risk, seeks to reduce risk factors in a society as a whole. Small changes made by the entire community may shift the mean values of risk factor prevalence and lead to a greater reduction in the incidence of coronary heart disease, at less cost per life saved than the "high risk" approach alone. ${ }^{711}$ But even with this approach extra effort may be needed among those groups in the population at added risk - that is, the socially disadvantaged. Because unhealthy lifestyles may be less entrenched in the young, children should be included in preventive programmes. $^{8}$

The difficulties of a population preventive approach have been well described by Rose and include a lack of clinical satisfaction (for doctors never know whether they really did anything for any particular patient) and the fact that few are enthused by the idea of making changes on behalf of the community in general - a "tax" on behaviour that may or may not yield a personal dividend. ${ }^{7}$ Furthermore, the political nature of attempts to redress outcomes of social inequality is unfamiliar or unacceptable territory for some medical practitioners.

None the less, the medical profession ought to weigh carefully the high risk and the population based approaches to prevention [see also $\mathrm{p}$ 1031]. If a policy based on concentrating 